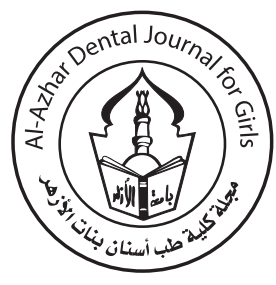

\title{
The Effect of Liner Placement under Bulk-Fill Resin Composite Restorations on Cuspal Deflection in Maxillary Premolar Teeth.
}

\begin{tabular}{l} 
Ahmed I. Bayoumi ${ }^{\mathbf{1}^{*}}$, Maha \\
\hline \begin{tabular}{l} 
Codex : 06/21.01 \\
azhardentj@ azhar.edu.eg \\
http://adjg.journals.ekb.eg \\
\hline DOI: 10.21608/adjg.2020.35680.1276 \\
\hline $\begin{array}{l}\text { Restorative Dentistry } \\
\text { (Removable Prosthodontics, Fixed } \\
\text { Prosthodontics, Endodontics, Dental } \\
\text { Biomaterials, Operative Dentistry) }\end{array}$ \\
\hline
\end{tabular}
\end{tabular}

\section{KEYWORDS}

Bulk-fill composites, Cuspal deflection, Universal microscope,

Activa Bioactive liner.

\begin{abstract}
Purpose: This study investigated the cuspal deflection in premolar teeth restored with different bulk-fill resin composites with or without using a liner. Materials and Methods: 60 extracted human upper premolar teeth were used. The samples in this study were divided into three main groups (20 samples for each) according to the materials used; Each group was subdivided into two subgroups according to presence of liner. Standardized large MOD cavities were prepared. The distance between two reference points on cusp tips was measured before the restoration using a universal microscope. Then the teeth were restored with different bulk-fill resin composites (Filtek bulk fill, X-tra fil bulk fill and Activa Bioactive Restorative Material) with and without a liner (Activa Bioactive base/ liner). Second reading of cuspal deflection was measured $15 \mathrm{~min}$ after the completion of restorations. All samples were subjected to thermal cycling using thermal cycler machine. Then the third reading of cuspal deflection was measured. The data were statistically analyzed with KolmogorovSmirnov and Shapiro-Wilk tests. Results: no statistically significant difference was found between the mean cuspal deflection of the three composites and cuspal deflection without liner showed statistically significantly higher mean value than with liner. Conclusion: The placement of Activa Bioactive base/liner beneath the bulk-fill resin composite restorations decreased cuspal deflection.
\end{abstract}

\section{INTRODUCTION}

Composite resin has a wide popularity among restorative materials as it provides high-quality esthetics and elongated time of fine clinical performance. The curing depth in composite resin is important when

- Paper extracted from Doctor thesis titled “Cuspal Deflection and Electromyographic Activity as well as Clinical Performance of Bulk-Fill Composite resins with Activa Bioactive Liner"

1. Assistant Lecture of Operative Dentistry Department, Faculty of Dentistry, Badr University, Cairo, Egypt.

2. Professor of Operative Dentistry, Faculty of Dental Medicine for Girls, Al-Azhar University, Cairo, Egypt.

3. Professor of Operative Dentistry, Faculty of Oral and Dental Medicine, Cairo University, Cairo, Egypt.

* Corresponding author email: ahmed.elbayoumy@buc.edu.eg 
taking the decision regarding the maximal increment thickness during restoration of teeth cavities. Conventional incremental technique is used to place direct composite filling, the composite is applied in several increments of a maximum thickness of $2 \mathrm{~mm}$ and the individual increment is light-cured separately for 10 to $40 \mathrm{~s}$, this depending on the light intensity of the curing light, the shade and degree of translucency of the composite material used.

The curing light, the shade and degree of translucency of the composite material used. This procedure could be a really long and method sensitive process, especially during its usage in filling huge cavities within the posterior region. As a result, most professionals were trying to find a practice-friendly substitute to this complicated multi-layer technique. Usage of a protocol which is more economic, offering increased reliability and safety and doesn't have a prolonged working time was their goal ${ }^{(1)}$.

Combination of a lowered method-sensitive and a confirmed clinical durability of amalgam along with the stability resulting from the procedure of adhesion are needed in a restorative material to allow efficiency in restoring posterior cavities. Materials are required to possess the following: Rapid and simple application, plain finishing and polishing, low technique sensitivity, more time-efficient and minimum procedures in selecting shades, preservation and stabilization of the natural tooth configuration and occurrence of the adhesion feature to tooth or not, or it just restricted negative effects on polymerization (Polymerization shrinkage and polymerization stress) ${ }^{(2)}$.

Bulk-fill composites were newly progressed due to the need for these properties to be present. Curability depth of this type of composite resins was found to be up to $4-5 \mathrm{~mm}$ thick, developing less value in polymerization shrinkage stresses and providing an increased value in the light transmission properties as a result of lessening the light distribution at the filler-matrix interface via one of the following: filler amount reduction or filler size enlargement. Achieving this huge curing depth happened when this restorative material was modified in its composition to maximize the penetrating ability of visible light throughout the materials such as enlarged filler size and novel photo-initiators ${ }^{(3)}$.

Nevertheless, the polymerization shrinkage presents the main disadvantage for direct composite resin due to the occurrence of stress build up leading to unpleasant results among the bonding process to tooth with consequences likes marginal staining, micro leakage, and recurrent caries. If the adhesive strength is more than the contraction stress, internal tension of the restoration is maintained and this result in the pulling of cavity walls jointly, thus intercuspal distance reduction (i.e., cuspal deflection). The amount of this inward cuspal movement is dependent to size of cavity and the type of composite used $^{(4)}$.

Enamel cracks, tooth fracture and post-operative sensitivity are the causes of cuspal deflection. Cuspal deflection extent is dependent on biomechanical factors such as: geometrical factors (including cavity dimensions and thickness of cavity walls after preparation) and different material Properties including (elastic modulus, polymerization shrinkage, flow and hygroscopic expansion of resin composite material), That signifies the first group. Whereas, the description of the second group is as: clinical factors including the technique of placement, direct or indirect restoration, usage of stress absorbing layers acquiring lessened elastic modulus liners, light curing unit and protocol ${ }^{(5)}$. Consequently, our study aims for the evaluation of how the liner placement under bulk-fill resin composite restorations affects cuspal deflection in maxillary premolars.

\section{MATERIAL AND METHODS}

A total of 60 freshly extracted maxillary human premolars were collected and examined for the presence of cracks or any other defects using eye loupe (4.5 x univet, Italy). Each tooth was marked 
with a maker at $2 \mathrm{~mm}$ apical to the cemento enamel junction. Teeth were embedded in Teflon ring (1.6 $\mathrm{cm}$ diameter and $2 \mathrm{~cm}$ height), containing chemically cured activated acrylic resin. The long axis of the tooth was adjusted parallel to that of the teflon ring and their occlusal surface parallel to plane of the acrylic mold and perpendicular to the plane of the base, using dental surveyor.

\section{Cavity preparation:}

Standardized MOD cavities (mesio-occlusaldistal) were prepared in all teeth with the desired cavity depth and width. The depth of the cavity at the occlusal isthmus was a standard of $(3.0 \mathrm{~mm})$ and positioning of all gingival walls was done on top of the amelocemental junction at the cervical aspect of the proximal boxes. The width of the floor was $3 \mathrm{mmand}$ axial height was $1 \mathrm{~mm}$. The cavity depth and width were checked using a graduated periodontal probe (fig 1).

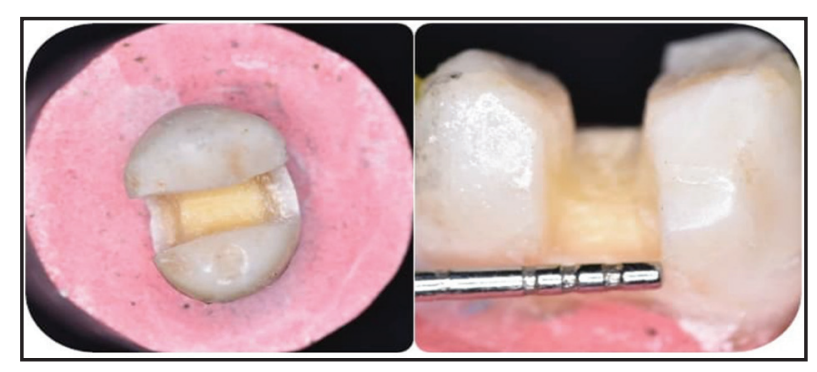

Figure (1): Occlusal isthmus was standardized to $3 \mathrm{~mm}$ width

\section{Sample grouping:}

The 60 prepared teeth were divided into three main groups according to types of composite material used (20 for each group), where the first group was restored by Activa Bioactive Restorative (PULPDENT), while the second group was restored by X-tra Fil bulk fill composite (VOCO) and the third group was restored by Filtek bulk fill composite (3M-ESPE). Each group was divided into two subgroups according to presence of Activa Bioactive Base/Liner (10 for each subgroup): the first subgroup had Activa Bioactive Base/Liner under composite, while the second subgroup with no Base/Liner. Presentation of composition and manufacture of materials used in this study is in table (1).

\section{Pre-restorative intercuspal distance measurements:}

Small shallow concavities were made with size 2 high speed round burs (Mani Inc, Japan) on the outer surface within the enamel at $1 \mathrm{~mm}$ below both cusp tips. Then two small glass balls $1.5 \mathrm{~mm}$ diameter were fixed to each cusp as reference points by using adhesive (fig 2) for intercuspal distance measurements by universal microscope (serial No.2510 Carl Zeiss-Germany) as it measured the distance between two glass balls. This was considered the intercuspal distance before restoration of cavities (baseline) (fig 2).

\section{Restorative procedure}

Half of the cavities were restored firstly by a thin layer of Activa Bioactive Base/liner of 1mm thickness horizontally on pulpal floor and cured for 20 seconds. Then the depth of the cavity was checked with periodontal probe after application of a liner and adjusted to $1 \mathrm{~mm}$. Then selective etching technique was used for all prepared cavities with or without liner. Acid etching (SpidentKorea) duration for enamel of prepared cavities was 30 seconds, rinsing duration was 30 seconds followed by drying. Then uses a self-etch (All bond universal, Bisco-USA) was apply to the entire cavity accordance with the manufacturer's instructions. The excess solvent was evaporated by gentle air drying for 5 seconds. The surface should maintain a uniform glossy appearance, and light cured for 20 seconds.

\section{Matrix application:}

Easy matrix (Tor VM) was used for MOD cavities. The matricing was performed while ensuring that the cuspal indices were not pressured. 
Table (1): Material's composition and manufacture:

\begin{tabular}{|l|l|l|l|}
\hline Materials and Brand & Specification & Manufacturer & Composition \\
\hline All Bond Universal & One step self etch & BISCO. USA & $\begin{array}{l}\text { Ethanol, water, 10-MDP, HEMA, and } \\
\text { initiators. }\end{array}$ \\
\hline $\begin{array}{l}\text { Filtek }{ }^{\text {TM }} \text { Bulk Fill } \\
\text { Posterior restorative }\end{array}$ & Nano-filled bulk fill composite & $\begin{array}{l}\text { 3M ESPE, } \\
\text { St.Paul. USA }\end{array}$ & $\begin{array}{l}\text { Fillers: aggregated silica/zirconia } \\
\text { nanoparticles, trifluoride filler, non- } \\
\text { agglomerated/ non-aggregated silica filler, } \\
\text { non-agglomerated /non aggregated zirconia } \\
\text { filler. } \\
\text { Matrix: Aromatic: AFM, Aromatic UDMA. }\end{array}$ \\
\hline $\begin{array}{l}\text { X-tra Fil } \\
\text { Posterior restorative }\end{array}$ & $\begin{array}{l}\text { Methacrylate bulk fill resin } \\
\text { composite light cured }\end{array}$ & $\begin{array}{l}\text { VOCO America, } \\
\text { Inc. USA }\end{array}$ & $\begin{array}{l}\text { Bis-GMA, UDMA-TEGDMA, Filllera } \\
\text { (Barium-Boron-Alumino-Silicate glass). }\end{array}$ \\
\hline $\begin{array}{l}\text { ACTIVA BioACTIVE } \\
\text { RESTORATIVE }\end{array}$ & $\begin{array}{l}\text { Composite resin that have a } \\
\text { higher bioactivity and releasing } \\
\text { higher fluoride than glass } \\
\text { ionomer }\end{array}$ & PULPDENT. USA & $\begin{array}{l}\text { Bioactive glass fillers, bioactive resin matrix, } \\
\text { shock absorbing resin component. }\end{array}$ \\
\hline $\begin{array}{l}\text { ACTIVA BioACTIVE } \\
\text { BASE/LINER }\end{array}$ & \begin{tabular}{l} 
Reactive glass fillers \\
\hline
\end{tabular} & PULPDENT. USA & $\begin{array}{l}\text { Sodium fluoride, mixture of methacrylates } \\
\text { and diurethane monomers with modified } \\
\text { polyacrylic acid. }\end{array}$ \\
\hline
\end{tabular}

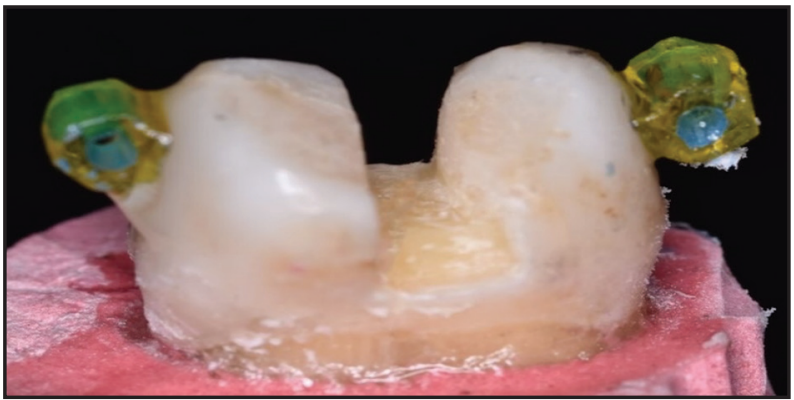

Figure (2): glass ball fixed to each cusp as reference points

\section{Bulk packing:}

All of the cavities were restored according to manufacturer's instructions in one large increment using., (A1: Activa Bioactive Restorative, A2: X-tra fil and A3: Filtek ${ }^{\mathrm{TM}}$ Bulk Fill,). Carving of the occlusal side among all restorations before curing was done for approximation of the regular occlusal anatomy, after curing the occlusal side for 40 seconds, Removal of easy matrix followed by light curing again from the two proximal surfaces for 20 seconds. Following completion of the restoring process, every tooth is retained damp via immersing it in water, air drying was then performed prior taking the second reading of intercuspal distance.

\section{Thermo cycling:}

All teeth were subjected to thermocycling with thermal cycler machine $\left(5^{\circ} \mathrm{C}\right.$ cold water and then in $55^{\circ} \mathrm{C}$ hot water for 5000 cycles), then the third reading for cuspal deflection was measured. The cuspal deflection was obtained by calculating the difference between final and the baseline measurements.

\section{Statistical analysis:}

Numerical data were explored for normality by checking the distribution of data and using tests of normality (Shapiro-Wilk and Kolmogorov-Smirnov tests). Cuspal deflection data showed normal (parametric) distribution. Presentation of data was as mean and standard deviation (SD) values. Repeated measures. Studying the consequence of composite type, liner, time and their interaction on mean cuspal deflection was done via Analysis of Variance (ANOVA). In case of ANOVA test's significance, Bonferroni's post-hoc test was done for pair-wise comparisons. 


\section{RESULTS}

\section{Effect of different interactions on cuspal deflection}

\section{a. Comparison between composite types:}

There was no statistically significant difference between the mean cuspal deflection values of the three composite types before, after polymerization as well as after thermocycling with and without liner.

Table (2): The mean, standard deviation (SD) values and results of repeated measures of ANOVA test for comparison between cuspal deflection of without and with liner with different interactions of variables.

\begin{tabular}{|c|c|c|c|c|c|c|c|}
\hline \multirow{2}{*}{ Composite type } & \multirow{2}{*}{ Time } & \multicolumn{2}{|c|}{ No liner } & \multicolumn{2}{|c|}{ Liner } & \multirow{2}{*}{$P$-value } & \multirow{2}{*}{$\begin{array}{c}\text { Effect size } \\
\text { (Partial eta squared) }\end{array}$} \\
\hline & & Mean & $\mathrm{SD}$ & Mean & SD & & \\
\hline \multirow{3}{*}{ Filtek-supreme } & Before polymerization & 13.162 & 0.43 & 12.962 & 0.618 & 0.388 & 0.014 \\
\hline & After polymerization & 13.018 & 0.348 & 12.810 & 0.542 & 0.367 & 0.015 \\
\hline & After thermocycling & 13.241 & 0.427 & 13.044 & 0.625 & 0.417 & 0.012 \\
\hline \multirow{3}{*}{ Xtra fil } & Before polymerization & 13.658 & 0.636 & 12.923 & 0.515 & $0.002 *$ & 0.159 \\
\hline & After polymerization & 13.483 & 0.733 & 12.87 & 0.527 & $0.010^{*}$ & 0.118 \\
\hline & After thermocycling & 13.759 & 0.635 & 13.226 & 0.656 & $0.031 *$ & 0.083 \\
\hline \multirow{3}{*}{ Activa bioactive } & Before polymerization & 13.165 & 0.427 & 13.146 & 0.408 & 0.935 & 0.0001 \\
\hline & After polymerization & 13.119 & 0.413 & 13.097 & 0.411 & 0.923 & 0.0001 \\
\hline & After thermocycling & 13.226 & 0.418 & 13.206 & 0.396 & 0.934 & 0.0001 \\
\hline
\end{tabular}

*: Significant at $P \leq 0.05$

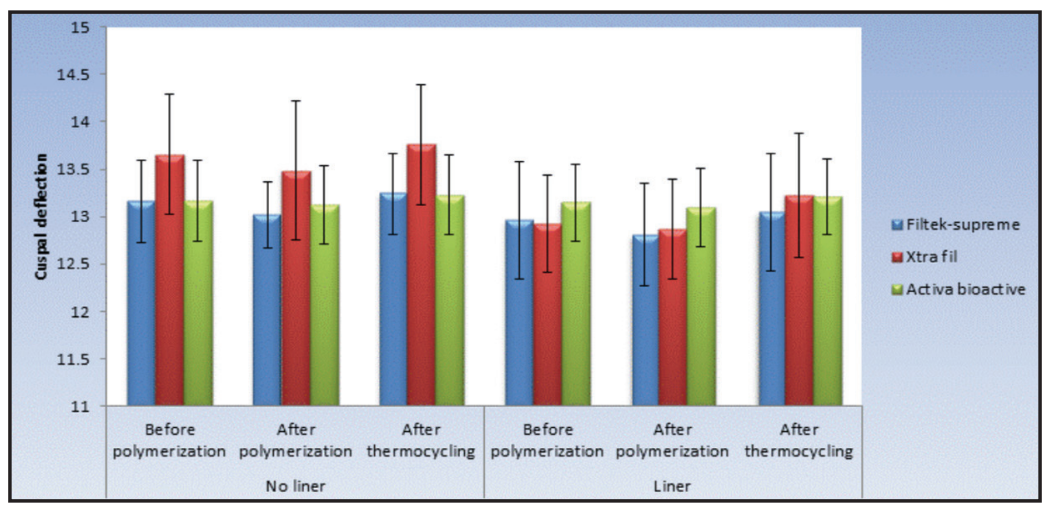

Figure (3): Bar chart representing mean and standard deviation values for cuspal deflection of the three composite types with different interactions of variables 


\section{b. Effect of liner regardless of composite type and time}

The mean cuspal deflection without liner showed statistically significantly higher mean value than with liner $(P$-value $=0.032)$.

Table (3): The mean, standard deviation (SD) values and results of repeated measures ANOVA test for comparison between cuspal deflections without and with liner regardless of composite type and time.

\begin{tabular}{|c|c|c|c|c|c|}
\hline \multicolumn{2}{|c|}{ No liner } & \multicolumn{2}{c|}{ Liner } & \multirow{2}{*}{ P-value } & $\begin{array}{c}\text { Effect size } \\
\text { (Partial eta } \\
\text { squared) }\end{array}$ \\
\hline Mean & SD & Mean & SD & & 0.083 \\
\hline 13.315 & 0.546 & 13.031 & 0.525 & $0.032 *$ & 0.03 \\
\hline
\end{tabular}

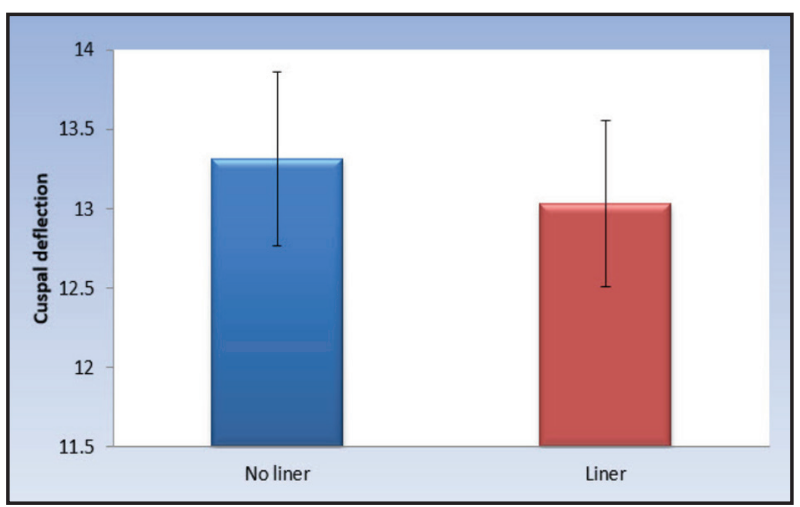

Figure (4): Bar chart representing mean and standard deviation values for cuspal deflections without and with liner regardless of composite type and time.

\section{c. Effect of time regardless of composite type and liner}

A statistically significant difference was found between mean cuspal deflections at different times of measurement ( $P$-value $<0.001)$. Pair-wise comparisons between times revealed that there was a statistically significant decrease in mean cuspal deflection after polymerization followed by a statistically significant increase in mean cuspal deflection after thermocycling. The mean cuspal deflection after thermocycling showed statistically significantly higher mean cuspal deflection than before polymerization value.
Table (4): The mean, standard deviation (SD) values and results of repeated measures ANOVA test for comparison between cuspal deflections at different times of measurement regardless of composite type and type.

\begin{tabular}{|c|c|c|c|c|c|c|c|}
\hline \multicolumn{2}{|c|}{$\begin{array}{c}\text { Before } \\
\text { polymerization }\end{array}$} & \multicolumn{2}{|c|}{$\begin{array}{c}\text { After } \\
\text { polymerization }\end{array}$} & \multicolumn{2}{c|}{$\begin{array}{c}\text { After } \\
\text { thermocycling }\end{array}$} & \multirow{2}{*}{ P-value } & $\begin{array}{c}\text { Effect size } \\
\text { (Partial } \\
\text { eta } \\
\text { squared) }\end{array}$ \\
\hline Mean & SD & Mean & SD & Mean & SD & & \\
\hline $13.169^{\mathrm{B}}$ & 0.548 & $13.066^{\mathrm{C}}$ & 0.536 & $13.284^{\mathrm{A}}$ & 0.562 & $<0.001^{*}$ & 0.260 \\
\hline
\end{tabular}

*: Significant at $P \leq 0.05$,

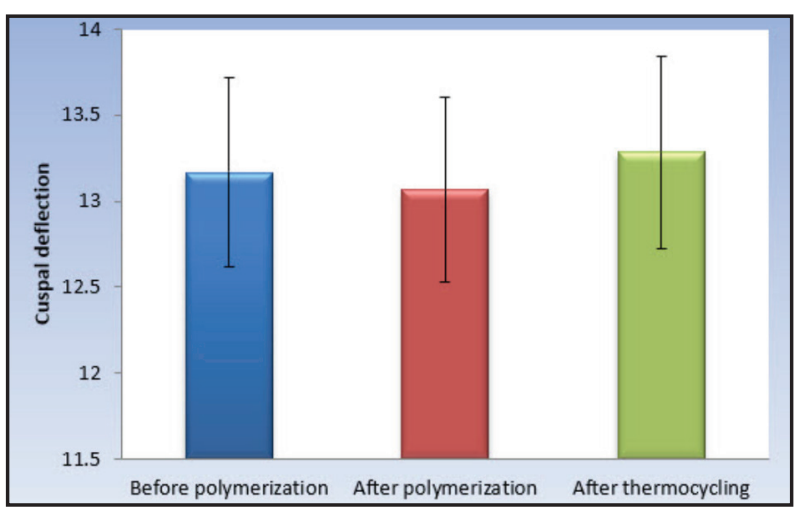

Figure (5): Bar chart representing mean and standard deviation values for cuspal deflections at different times regardless of composite type and liner

\section{DISCUSSION}

Cuspal deflection is considered a frequent complication that happens due to polymerization stress of composite on tooth structure. It behaves like a preloading that makes fracture process of the tooth easier when it is subjected to tension; this is considered the chief reason of failure in composite curing ${ }^{(6)}$.

Extracted teeth are now widely used in measuring cuspal deflection as they removed the conformity of the testing system and supporting structures. The inclusion of maxillary premolar teeth in this study is due to that they are constant in size and shape ${ }^{(3)}$. 
Preparation of Large MOD cavities in this study as it favors potential cuspal deflection, a bigger cavity size lead to increase cuspal deflection. Explanation of this is as follows: firstly, a smaller amount of tooth structure remains in large cavities, and that causes added flexibility of the cusps and more conformity with composite shrinkage. If the cavity is left wider and deeper during preparation, the strength of the prepared tooth is significantly lowered and flexibility is increased. Secondly, the more the overall volume of composite required for restoring large cavities causes elevated shrinkage force ${ }^{(5)}$.

The distance between the two reference points was calculated following 15 minutes since completion of restoring process as the cuspal deflection was more slower and longer than polymerization shrinkage of the composites and numerous studies concluded that, the greatest quantity of inward displacement happened during this time and the specimens were in complete hydration, the residual free radicals may have been the result. Persistence of Double bonds' reaction within resin base restoration has occurred. As a result, the deformation was continuous for quite a lot of minutes following complete polymerization procedure $^{(6)}$.

So, bulk fill composites (X-tra fil, Filtek ${ }^{\mathrm{TM}}$ bulk fill and Activa BioACTIVE Restorative) were chosen in this study because they have excellent cavity adaptation, one-step placement up to $4 \mathrm{~mm}$, excellent handling and sculptability as claimed by manufacturer. Moreover, they demonstrated ease to use and low polymerization shrinkage rates to reduce cuspal deflection. Thus, results of the present study revealed that no significant differences among all experimental groups was found when the displacement was measured after cavity preparation. These results are in accordance with other studies that reported that the teeth were exposed to deformation after cavity preparation but this deflection was not statistically significant among all groups because they were performed by same operator and prepared by the standardized method ${ }^{(7)}$.
As, samples restored with Activa BioActive restorative showed mean value of cuspal deflection after restoration (mean=13.16 \pm 0.397 ). This might be attributed to the fact that, it contains a rubberized resin component that makes them tougher and more resistant to fracture and chipping than composites in addition to the absence of Bisphenol A, or Bis-GMA or BPA derivatives ${ }^{(8)}$. While, X-tra fil composite recorded a mean value of cuspal deflection of (13.32 \pm 0.688$)$, this may be ascribed to volumetric polymerization shrinkage of X-tra fil $(1.7 \%)$, the percentage of fillers present which was found to be $86 \%$ by weight and $70 \%$ by volume as mentioned by their manufacturers. Besides, modulus of elasticity of X-tra fil $16 \mathrm{GPa}$ which is a momentous aspect in stress development ${ }^{(3)}$.

Moreover, the Filtek bulk fill showed mean value of cuspal deflection $(13.28 \pm 0.708)$ subsequent to restoration, this could be the result of the elimination of the monomer TEGDMA (286 g/mol) out of its resin system. Furthermore, Filtek Bulk Fill possesses two innovated monomers that are responsible for a main function in minimizing shrinkage stress as AUDMA and AFM. Fragmentation chain-transfer property was found in AFM; the benefit of addition fragmentation chain-transfer is due to the fact that it enhances the adaptability of the covalent network to stress creation by means of bond breakage and restructuring, with nonexistent net loss of crosslinking via an allyl disulfide bond ${ }^{(9)}$.

In this study, regardless of composite types and time; the mean cuspal deflection without liner showed statistically significant higher mean value than with liner $(P$-value $=0.032)$. This might be attributed to the fact that, the Activa Bioactive base/ liner has a strong, resilient resin matrix that doesn't induce chipping or crumbling and using Activa Bioactive liner could result in reduction in the composite's volume required to effective restoring ensuing lesser value of shrinkage force and polymerization shrinkage stresses. Also, Activa is the primary bioactive base/liner having resin matrix in ionic form, a shock-absorbing resin component 
and Bioactive fillers which imitate both chemical and physical properties of natural teeth ${ }^{(10)}$.

These results are supported by other researches which proved that the amount of cuspal deflection was significantly decreased in MOD cavity when the usage a base material RMGIC compared to those cavities restored with composite resin only ${ }^{(11)}$. Furthermore, it was found that, the (resin modified glass ionomer cement) intermediate layer caused major diminish in polymerization contraction force values of the restorative system ${ }^{(12)}$.

Moreover, during the use of the flowable bulk-fil resin based composite (RBC) as a base in restoring MOD cavities under incremental restoration demonstrated significantly reduced cuspal deflection compared with the oblique incremental filling technique only, suggesting the possibility of treatment of MOD cavity as a modified cantilever beam , a scenario in which the deformation equals a cubed power of the length. Therefore, decreased in the length of cusp, considerably lowers the deflection in a way comparable to when a resin-modified glassionomer is positioned underneath a $\mathrm{RBC}^{(1)}$.

On the other hand, these results were in contradiction with another study that claimed that, placing glass ionomer wasn't of a significant value in reduction of the total cuspal deflection. This could be attributed to material and technique different ${ }^{(13)}$. Moreover, another study revealed that the highest cuspal deflection values were recorded with nanohybrid restorations lined with nano-flowable resin composite which did not different significantly than those without liner. Which due to its high bond strength with tooth structure. The high deflection values may be explained by the higher contraction stresses resulting from polymerization reaction of nanohybrid resin composite and low filler content together with high volumetric polymerization shrinkage of flowable composite resin ${ }^{(5)}$.

There was a statistically significant decrease in mean cuspal deflection after polymerization followed by a statistically significant increase in mean cuspal deflection after thermocycling. The mean cuspal deflection after thermocycling showed statistically significantly higher mean cuspal deflection than before polymerization value.

This is in agreement with other study that reported, after water storage, cuspal deflection was highly significantly different with restorative materials. This could be related to the fact that; the composite restoration presents some stress relaxation assignable to water absorption. The viscoelastic properties of composites may be the cause of stress relaxation, whereas water absorption would add to relaxation by the means of hydrolysis (degradation of the polymers chemically) and plasticization (water induced molecular mobility) effects ${ }^{(14)}$.

\section{CONCLUSIONS}

Under the conditions of the current study, the following conclusions could be delivered:

- The amount, type and size of the filler are crucial factors in determining the amount of cuspal deflection.

- Presence of liner appears beneficial in reducingcuspal deflection.

- Bulk-fill resin composites generated comparable cuspal deflection behavoir.

\section{REFERENCES}

1. Zahraa AJ and Zainab MA. Evaluation of the cuspal deflection of premolars restored with different types of bulk fill composite restorations (A comparative in vitrostudy). Biomed \& Pharmacol. J. 2018; 11: 751-7.

2. Demirel G, Baltacioglu IH, Kolsuz ME, Ocak M, Bilecenoglu B and Orhan K. Volumetric cuspal deflection of premolars restored with different paste-like bulk-fill resin composites evaluated by microcomputed tomography. Oper Dent.2020:45:143-50.

3. Haytham B, Omar E, Wafa E, Belal S and Sameh N. Cuspal deflection of premolars restored with bulk-fill composite resins. Esthet and Rest Dent J. 2016; 122-30. 
4. Rebecca E, Mathias S, Kirstin Vand Petra H. Influence of delayed light curing on the cuspal deflection and microleakage of a dual-cured composite resin in class 2 restorations. Quintessence Int.2019;50:94-102.

5. Mohammed SA and Nashaat M. Cuspal deflection and fracture resistance in maxillary premolar teeth restored with bulk-fill flowable resin-based composite materials. International Journal of Health Sciences \& Research. 2018;8: 105-12.

6. Akimasa T, Yuko N, Wayne WB, Hidehiko W, William WJ, Toshiki et al. Simulated cuspal deflection and flexural properties of high viscosity bulk-fill and conventional resin composites. Journal of the Mechanical Behavior of Biomedical Materials. 2018;87: 111-18.

7. Politi, McHugh LEJ, Al-Fodeh RS and Fleming GJP. Modification of the restoration protocol for resin-based composite (RBC) restoratives (conventional and bulk fill) on cuspal movement and microleakage score in molar teeth. Dental Materials.2018; 1-7.

8. Barry M. Owens, Jeffrey GP and William W. Johnson. Evaluation of the marginal integrity of a bioactive restorative material. General Dentistry :2018; 32-6.
9. Fugolin, APP, and Pfeifer CS. "New Resins for Dental Composites.” J Dent research; 2017:96: 1085-91.

10. Annelies VE, Jan DM, Diogo PL and Bart VM. Bulk-Fill composites: A review of the current literature. J Adhes Dent; 2017:19.95-109.

11. Fatma D, Esra E and Sevil G. Comparison of different base materials on fracture strength of mesio-occlusal-distal composite restorations. Eur J Gen Dent.2018;7:25-30.

12. Agarwal PM, Taneja S and Kumar M. To evaluate and compare the effect of different light-curing modes and different liners on cuspal deflection in premolar teeth restored with bulk filled or incrementally filled composite measured at different time intervals. J Conserv Dent. 2017:20:317-32.

13. Nguyen KV, Wong RH, Palamara J and Burrow MF. The Effect of Resin-modified glass-ionomer cement base and bulk-fill resin composite on cuspal deformation. Oper Dent. 2016; 41: 208-18.

14. Shahenda S, Usama MA, Ali A, Hend E, Alireza V and Ikuya W. Shrinkage stress of high and low viscosity bulkfill composites with incremental and bulk fill techniques. Tanta Dent J.2018:15:224-33. 